\title{
The research of $(2,1)$-total labelling of trees basen on Frequency Channel Assignment problem
}

\author{
Haina Sun ${ }^{1, a}$ \\ ${ }^{1}$ Department of Fundamental Courses, Ningbo Institute of Technology, Zhejiang Univ. Ningbo 315100, China
}

\begin{abstract}
Let $\mathrm{T}$ be a tree, Let $D_{\Delta}(T)$ denote the set of integers $\mathrm{k}$ for which there exist two distinct vertices of maximum degree of distance at $\mathrm{k}$ in $\mathrm{T}$. The $(2,1)$-total labelling number of a graph $\mathrm{G}$ is the width of the smallest range of integers that suffices to label the vertices and the edges of $G$ such that no two adjacent vertices have the same label, no two adjacent edges have the same label and the difference between the labels of a vertex and its incident edges is at least 2. In this paper, we prove that if $\mathrm{T}$ is a tree with $\Delta \geq 5$ and $3,4 \notin D_{\Delta}(T)$, then $\mathrm{T}$ is Type 1 .
\end{abstract}

\section{Introduction}

Motivated by the Frequency Channel Assignment problem, Griggs and Yeh[1]introduced the $L(2,1)$ labelling of graphs. This notion was subsequently generalized to the $L(p, q)$-labelling problem of graphs. Let $p$ and $q$ be two nonnegative integers. An $L(p, q)$ labelling of a graph $G$ is a function $f$ from its vertex set $V(G) \quad$ to the set $\{0,1, \cdots, k\}$ such that $|f(x)-f(y)| \geq p$ if $x$ and $y$ are adjacent, and $|f(x)-f(y)| \geq q$ if $x$ and $y$ are at distance 2 . The $L(p, q)$-labelling number $\lambda_{p, q}(G)$ of $G$ is the smallest $k$ such that $G$ has an $L(p, q)$-labelling $f$ with $\max \{f(v) v \in V(G)\}=k$.In particular, we simply write $\lambda(G)=\lambda_{2,1}(G)$. The $L(p, q)$-labelling of graphs have been studied rather extensively in recent years $[2,3,4,5,6]$. Whittlesey, Georges and Mauro [7] investigated the $L(2,1)$-labelling of incidence graphs. The incidence graph of a graph $G$ is the graph obtained from $G$ by replacing each edge by a path of length 2 . The $L(2,1)$-labelling of the incidence graph of $G$ is equivalent to an assignment of integers to each element of $V(G) \cup E(G)$ such that adjacent vertices have different labels, adjacent edges have different labels, and incident vertex and edge have the difference of labels by at least 2. This labelling is called $(2,1)$-total labelling of graphs, which was introduced by Havet and $\mathrm{Yu}$ [8] and generalized to $(d, 1)$-total labelling. Let $d \geq 1$ be an integer. A $k-(d, 1)$-total labelling of a graph $G$ is a function $f$ from $V(G) \bigcup E(G)$ to the set $\{0,1, \cdots, k\}$ such that $f(u) \neq f(v)$ if $u$ and $v$ are two adjacent vertices, $f(e) \neq f\left(e^{\prime}\right)$ if $e$ and $e^{\prime}$ are two adjacent edges, and $|f(u)-f(e)| \geq d$ if a vertex $u$ is incident to an edge $e$.

Let $\Delta(G)$ (or simply $\Delta$ ) denote the maximum degree of a graph $G$. Suppose that $T$ is a tree with $\Delta \geq 3$. It is easy to prove that $\Delta+1 \leq \lambda(T) \leq \Delta+2$ and $\Delta+1 \leq \lambda_{2}^{t}(T) \leq \Delta+2$. Wang [9] showed that if $T$ does not contain two vertices of maximum degree at distance either 1, 2, or 4, then $\lambda(T)=\Delta+1$. Moreover, examples of trees $T$ having two vertices of maximum degree at distance 4 such that $\lambda(T)=\Delta+2$ was constructed in [10].

A star is a tree that consists of $\Delta$ leaves (A leaf is a $1-$ vertex) and a $\Delta-$ vertex. A generalized star is a tree that all vertices are leaves except that two adjacent vertices.A star is also a generalized star, the $L(2,1)$ - total labelling number of a generalized star is $\Delta+1$. A tree $T$ is called Type 1 if $\lambda_{2}^{t}(T)=\Delta+1$ and Type 2 if $\lambda_{2}^{t}(T)=\Delta+2$. Let $M$ denote a generalized star, a tree of order 8 consisting two adjacent 4 -vertices and four leaves. Let $K_{1,4}$ denote the star of order 5 . Clearly, both $K_{1,4}$ and $M$ are Type 1 .

Let $T$ be a tree with $\Delta \geq 3$. We use $|T|$ to denote the number of vertices of $T$, and $d(v)$ to denote the degree of a vertex $v$ in $T$. A vertex of degree $k$ is called a $k$ vertex. The vertex $v$ is called major if $d(v)=\Delta$, minor

\footnotetext{
${ }^{\mathrm{a}}$ Corresponding author: shn@nit.zju.edu.cn
} 
if $d(v)<\Delta$, a leaf if $d(v)=1$, and a handle if $d(v)>1$ and $v$ is adjacent to at most one vertex of degree greater than one. A $k$-handle is a handle of degree $k$. A major handle is a vertex of maxi- mum degree that is a handle. Obviously, every tree $T$ with $|T| \geq 3$ that is not a star contains at least two handles. Let $\ell(P)$ denote the length of a path $P$. The distance between two vertices $x$ and $y$ in $T$ is the length of a shortest path connecting them. The diameter, denoted by $\operatorname{diam}(T)$, of $T$ is the maximum distance between two vertices of $T$. Let $D_{\Delta}(T)$ denote the set of integers $k$ for which there exist two distinct major vertices of distance at $k$ in $T$.

A generalized star is a tree $T$ with at least $|T|-2$ leaves. Obviously, the star $K_{1, n}$ is a special generalized star, which has $n=\left|K_{1, n}\right|-1$ leaves. For a vertex $u \in V(T)$, we use $L(u)$ to denote the set of leaves which adjacent to $u$. For $S \subseteq V(G)$, let $G-S$ denote the graph obtained from $G$ by deleting all the vertices in $S$. Also a tree $T$ is called Type 1 if $\lambda_{3}^{t}(T)=\Delta+3$ and Type 2 if $\lambda_{3}^{t}(T)=\Delta+4$.

Given an edge $e=v u \in E(T)$, we use $T_{v}(e)$ to represent the subtree of $T$ which is rooted at the vertex $v$ and contains the edge $e$.

Lemma 1 [11]. Let $T$ be a tree with $\Delta=4$ that is not a generalized star. If $3,4 \notin D_{\Delta}(T)$, then $T$ contains one of the following configurations:

(C1) A leaf $u$ is adjacent to a minor vertex $v$.

(C2) A path $x_{1} x_{2} x_{3} x_{4}$ such that $d\left(x_{2}\right)=2$ and $x_{1}$ is a major handle.

(C3) A path $x_{1} x_{2} x_{3} x_{4}$ such that $d\left(x_{2}\right)=3, x_{1}$ is a major handle and $x_{2} y_{1} y_{2}$ is a path, where $y_{1}$ is a neighbor of $x_{2}$ with $y_{1}$ is a major handle. $\operatorname{deg}\left(x_{3}\right)=2$ or 4 .

(C4) A path $x_{1} x_{2} x_{3} x_{4}$ such that $x_{1}$ is a major handle and $x_{2}$ is a weak major handle.

(C5) A path $x_{1} x_{2} x_{3} x_{4}$ such that $x_{1}$ is a major handle and $x_{2}$ is $\Delta$-degree, $x_{2} y_{1} y_{2}$ is a path, where $y_{1}$ is a neighbor of $x_{2}$ with $y_{1}$ is a major handle, the neighbor of $x_{2}$ other than $x_{1}, x_{3}$ and $y_{1}$ is a leaf.

Lemma 2 [11]. Let $T$ be a tree with $\Delta \geq 5$ that is not a star. If $3,4 \notin D_{\Delta}(T)$, then $T$ contains one of the following configurations:

(B1) A leaf is adjacent to a minor vertex.
(B2) A minor vertex $v$ is adjacent to $d(v)-1$ major handles.

(B3) A major handle is adjacent to a weak major handle.

(B4) A path $x_{1} x_{2} x_{3} x_{4}$ such that $x_{1}$ is a major handle and $x_{2}$ is $\Delta$-degree, $x_{2}$ adjacent to $\Delta-2$ major handles at most.

\section{Conclusion}

Theorem 3. If $T$ is a tree with $\Delta \geq 5$ and $3,4 \notin D_{\Delta}$, then $T$ is Type 1.

Proof The proof is proceeded by induction on $|T|$. The theorem holds clearly if $|T|=5$. Let $T$ be a tree with $|T| \geq 6, \Delta \geq 5$ and $3,4 \notin D_{\Delta}(T)$. If $T$ is a generalized star, it is easy to construct a $(2,1)$-total labelling of $T$ using the label set $B=\{0,1, \cdots, \Delta+1\}$. Thus, assume that $T$ is not a generalized star. We need to diverse the following four cases.

If $T$ contains (B1), then $T$ contains a leaf $x_{1}$ adjacent to a minor vertex $x_{2}$, then $T-x_{1}$ has a $(2,1)$ total labelling $f$ using $B=\{0,1, \cdots, \Delta+1\}$ by the induction hypothesis. Since $d\left(x_{2}\right) \leq \Delta-1$, there exist at most $\Delta-2+3=\Delta+1$ forbidden labels for the edge $x_{1} \quad x_{2}$ and at most four forbidden labels for the vertex $x_{1}$. By $|B|=\Delta+2$, we can first extend $f$ to $x_{1} x_{2}$ and then to $x_{1}$. Hence, assume that no leaf is adjacent to a minor vertex.

If $T$ contains (B2), then $T$ contains a configuration: , a minor vertex a adjacent to $d(a)-1$ major handles $x_{1}, x_{2}, \cdots, x_{d(a)-1}$ and the other vertex $b$. Let

$T^{\prime}=T-\bigcup_{i=1}^{d(a)-1}\left(L\left(x_{i}\right) \cup\left\{x_{i}\right\}\right)$.

By the induction hypothesis, $T^{\prime}$ has a $(2,1)$-total labelling $f$ using $B=\{0, \cdots, \Delta+1\}$.

If $f(a) \notin\{0, \Delta+1\}$, then $f$ can be extended into a (2,1) -total labelling of $T$. Assume that $f(a) \in\{0, \Delta+1\}$. Relabel a with a label from.

$$
\begin{aligned}
& B-\{0, \Delta+1, f(b), f(a b)-1, f(a b), f(a b)+1\} \\
& \quad \text { Since } \\
& \quad|B-\{0, \Delta+1, f(b), f(a b)-1, f(a b), f(a b)+1\}| \\
& \geq|B|-6=\Delta+2-6 \geq 5+2-6=1, \text { such a } \\
& \text { relabelling is feasible, } f \text { can be extended to } T .
\end{aligned}
$$


If $T$ contains (B3): A major handle is adjacent to a weak major handle. We let $x_{1}$ be a major handle and $x_{2}$ a weak major handle. If $f\left(x_{2}\right)=0$, we relable $x_{1}, x_{1} x_{2}$ with 5,3. If $f\left(x_{2}\right)=5$, we relable $x_{1}, x_{1} x_{2}$ with 0,2 . Because the other adjacent vertices of $x_{1}$ are leaves, we can easily conclude that T is Type 1 .

If $T$ contains (B4): A path $x_{1} x_{2} x_{3} x_{4}$ such that $x_{1}$ is a major handle and $x_{2}$ is $\Delta$ degree, $x_{2}$ adjacent to $\Delta-2$ major handles at most. We let

$$
T^{\prime}=T-\left\{x_{1}\right\}-L\left(x_{1}\right)+x_{2}+a
$$

Where $a$ is a leaf. By the induction hypothesis, $T^{\prime}$ has a (2,1)-total labelling. Because in $T^{\prime}, f\left(x_{2}\right)=0$ or 5 , so we diverse the following two cases:

If $f\left(x_{2}\right)=0$, then we exchange $a$ with $x_{1}$, we label $x_{1} x_{2}$ with label in $B-\left\{0,1, f\left(x_{2} u_{1}\right), f\left(x_{2} u_{2}\right),---\right.$, $f\left(x_{2} u_{\Delta-2}\right)$, Where $u_{1}, u_{2,---,} u_{\Delta-2}$ are the adjacent vertices of $x_{2}$ and different from $x_{1}$ and $x_{3}$. Since

$\mid B-\left\{0,1, f\left(x_{2} u_{1}\right), f\left(x_{2} u_{2}\right),---\right.$

$\left.f\left(x_{2} u_{\Delta-2}\right)\right\} \mid \geq \Delta+1-\Delta=1$,

so this label is feasible. Then we label $x_{1}$ with 5 .

If $f\left(x_{2}\right)=5$, we use the same method. We exchange $a$ with $x_{1}$, we label $x_{1} x_{2}$ with label in $B$ $\left\{0,1, f\left(x_{2} u_{1}\right), f\left(x_{2} u_{2}\right),---, f\left(x_{2} u_{\Delta-2}\right)\right\}$, Where $u_{1}, u_{2,---,} u_{\Delta-2}$ are the adjacent vertices of $x_{2}$ and different from $x_{1}$ and $x_{3}$. Since

$B-\left\{0,1, f\left(x_{2} u_{1}\right), f\left(x_{2} u_{2}\right),---\right.$,

$\left.f\left(x_{2} u_{\Delta-2}\right)\right\} \mid \geq \Delta+1-\Delta=1$,

so this label is feasible. Then we label $x_{1}$ with 0 .

\section{ACKNOWLEDGEMENTS}

This article is supported by Natural Science Foundation of Ningbo City (under) Grant 2013A610067.

\section{REFERENCES}

1. W. K. Hale: Frequency assignment: theory and applications, Proc IEEE 68(1980), p.1497-1514.
2. J. R. Griggs and R. K. Yeh: Labelling graphs with a condition at distance 2, SIAM J Discrete Math 5(1992), p.586-595.

3. G. J. Chang and D. Kuo: The labelling problem on graphs, SIAM J Discrete Math 9(1996), p. 309-316.

4. G. J. Chang, W.-T. Ke, D. Kuo, Daphne D.-F. Liu, and R. K. Yeh: On labelling of graphs, Discrete Math 220(2000), p. 57-66.

5. J. P. Georges, D. W. Mauro, and M. I. Stein: Labeling products of complete graphs with a condition at distance two, SIAM J Discrete Math 14(2000), p. 28-35.

6. D. Kr'al and R. 'Skrekovski: A theorem about the channel assignment problem, SIAM J Discrete Math 16(2003), p.426-437.

7. M. Molloy and M. R. Salavatipour: A bound on the chromatic number of the square of a planar graph, $\mathrm{J}$ Combin Theory Ser B 94(2005), p.189-213.

8. D. Sakai: Labelling chordal graphs: distance two condition, SIAM J Discrete Math 7(1994), p.133140.

9. F.Bazzaroa,M. Montassier, and A. Raspaud: total labelling of planar graphs with large girth and high maximum degree, Discrete Math, in press.

10. D. Chen and W. Wang, total labelling of outerplanar graphs, submitted.

11. Haina Sun, Trees with 3,4 are not in maximum degree distance, Lecture Notes in Information Technology, 3(2011),p. 91-95 